\title{
Iris Recognition System Using ANFIS Classifier
}

\author{
Neelam Sharma ${ }^{1}$, Preeti Singh ${ }^{2}$ \\ ${ }^{1}$ Computer Science \& Engineering, Vindhya Institute of Technology \& Science, Satna, M.P., India \\ ${ }^{2}$ Computer Science \& Engineering, Vindhya Institute of Technology \& Science, Satna, M.P., India
}

\begin{abstract}
A biometric system provides automatic identification of a character based on a unique feature or attribute possessed by the creature. Most practical iris recognition systems use original algorithms developed by Daugman and these algorithms are competent to create perfect recognition rates but not includes time requirement in account and extremely suffered when pupil is illuminated with light intensity. For the efficient pupil detection a morphological bridged canny edge detection technique is proposed, which is especially designed to handle pupil occlusion problem. For the feature selection and mining part, most conventional iris recognition systems relies on wavelet features extracted from rubber sheet model, but the wavelet feature extraction is a complex and time consuming process, to handle this problem in this paper rubber sheet model of iris part is exclusively used as feature. The third and most crucial modification proposed in this paper, is in the feature classification part, after feature space formation using rubber sheet model, ANFIS classifier is used for recognition efficiency.
\end{abstract}

Keywords: Pupil detection, Canny edge detection, Daugman rubber sheet model, Feature extraction

\section{Introduction}

The idea of mistreatment iris patterns for private identification was originally projected by Associate in Nursing eye doctor named Frank Burch in 1936 [9]. In 1980's the concept had appeared in films, however it still remained fantasy and conjecture. In 1987 two different ophthalmologists, Aran Safir and author Flom, proprietary this plan, and in 1989 they asked John Daugman (then teaching at Harvard University) to form actual applicable algorithms for iris recognition.

The Daugman algorithms can be found at the start within the original paper "High confidence visual recognition of persons by applied mathematics independence." They combined the field of classical pattern recognition with fashionable pc vision, mathematical statistics and studies of the human-machine interface. It's Ass ociate in nursing knowledge base field. The patents area unit in hand by Iridian Technologies and area unit the idea for all current iris recognition systems and product [9]. This identification system is been used in United Arab Emirates (UAE) for border-crossing management and as at 2004, it had been in a position to generate 420,000 expellees from a hundred and eighty nations within the world [10].

A biometric system provides automatic recognition of associate degree individual primarily based on some kind of distinctive feature or characteristic possessed by the individual. Biometric systems are developed supported fingerprints, countenance, voice, hand pure mathematics, handwriting, the tissue layer and also the one conferred during this thesis, the iris Biometric systems work by first capturing a sample of the feature, like recording a digital sound signal for voice recognition, or taking a digital color image for face recognition.

The iris is perforated near its center by a circular aperture called the pupil. The function of the iris is to control the amount of light entering through the pupil, and this can be done by sphincter and the dilator muscle and the dilator muscles alters the size of the pupil. The common diameter of the iris is $12 \mathrm{~mm}$, and therefore the pupil size will vary from $10 \%$ to $80 \%$ of the iris Diameter [2]. Biometrics deals with automatic strategies of recognizing someone primarily based on physiological characteristics such as face, fingerprints, hand pure mathematics, iris, retinal, and vein. Identity verification technique supported iris patterns is appropriate for prime level security systems. Iris is that the rounded ring between the pupil and also the albuginea of the attention. The structure of iris is mounted from concerning one year in age and remains constant over time. It exhibits long-run stability and infrequent re-enrolment necessities. The variations within the grey level intensity values distinguish two people.

\section{Background and Prior Research}

Different iris recognition ways that aid associate acceptable outlook for future work to build integrated classifier on latest input devices for glorious business transactions are mentioned. Benchmark databases, merchandise also are mentioned. Since the world is presently one amongst the foremost on the go and also the bulk of analysis is extremely giant, this survey covers a number of ways. The Strength of iris recognition systems over different statistics systems in security applications were initial known Description and limitations of the general public iris databases that square measure want to check the performance of those iris recognition algorithms was conjointly given. Several of the already developed systems couldn't handily work for black iris, modification of existing system is important for them to acknowledge black iris properly. A Literature Review on Iris Segmentation Techniques for Iris Recognition Systems because the part of work of iris segmentation has its own major applications in the field of police investigation as well as in security functions. The performance of the iris recognition systems depends heavily on segmentation and standardization techniques. A review of assorted segmentation approaches employed in iris recognition is finished. A biometric technique utilized in position applications like border crossing systems, single issue identification for the ATM users, India's Aadhaar project, landing field security. Iris recognition is one in all simplest 


\section{International Journal of Science and Research (IJSR) \\ ISSN (Online): 2319-7064}

Index Copernicus Value (2015): 78.96 | Impact Factor (2015): 6.391

methodology for identification of creature. Most of the time for iris segmentation and localize, wavelets area unit effective resolution and for committal to writing purpose physicist filters area unit used.

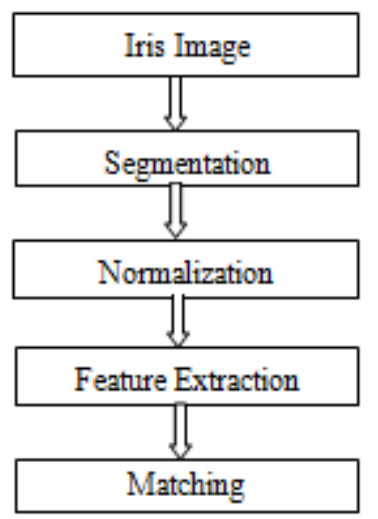

Figure 1: IRIS Recognition Systems.

\section{A. Iris Image}

Biometrics deals with automatic strategies of recognizing someone primarily based on physiological characteristics such as face, fingerprints, hand pure mathematics, iris, retinal, and vein. Identity verification technique supported iris patterns is appropriate for prime level security systems.

\section{B. Segmentation}

A technique is needed to isolate and exclude the artifacts as well as locating the circular iris region. The inner and also the outer boundaries of the iris are calculated.

a. Hough Transform: - The Hough rework is a commonplace pc vision algorithmic program which will be wont to verify the of easy parameters geometric objects, like lines and circles, gift in a picture. The circular Hough rework will be used to deduce the radius and center coordinates of the pupil and iris regions. Associate degree automatic segmentation algorithmic program supported the circular Hough rework is utilized by Wildes.

\section{Normalization}

Iris of all is completely different of us and will be captured in several sizes, for identical person put together in size might vary as a result of the variation in illumination and various factors. The group action technique will end up iris regions that have identical constant dimensions, so that two pictures of identical iris at a lower place all completely different conditions will have characteristic choices at identical special location. In general association rule mining can be thought of as a two step process.

(a) Generating all item sets having support greater than or equal to the user defined minimum support.

(b) Generate all the rules having the confidence factor greater than or equal to the user defined minimum confidence.

\section{Feature Extraction}

The significant choices of the iris ought to be encoded so as that comparisons between templates square measure usually created. Most iris recognition systems build use of a band pass decomposition of the iris image to form a biometric model. Iris provides texture data. A feature vector is designed that consists of the ordered sequence of choices extracted from the numerous illustration of the iris footage. Feature writing identifies the foremost necessary feature for categorization and for proper recognition of personals; many iris image ought to be extracted in a very precise manner.

\section{E. Matching}

To manifest via identification (one-to-many guide matching) or verification (one to - one guide matching), a guide created by imaging the iris is compared to a hold on price guide during various information. If the playing distance is below the decision threshold, identification has effectively been created e.g. A playing distance of zero would finish in a perfect match. Once generate of iris code, got to be compelled to match this iris guide with hold on guide in info throughout enrollment and see if any matches happens.

\section{Problem Formulation}

In this section, we formally define the research problem. First, my work proposes a highly efficient real time iris recognition system based on simplest feature space of rubber sheet models discrete wavelet transform (DWT) feature, along with the efficient ANFIS classifier for iris image classification. The identified problems involve in traditional iris recognition system are:

1) The process is much complex and not found suitable for real time recognition.

2) In the first step, that is for iris segmentation part mostly canny edge detector with Hough transform for circle detection has been employed. This process fails to avoid iris occlusion problem and not to draw complete circle and hence provides wrong information about pupil center coordinates and pupil radius.

3) In case of traditional system, lots of complex algorithms have been proposed by the researchers to extract features of the iris for recognition process, most of the time all these methods were very time consuming; hence an algorithm is required to overcome this problem.

4) The last but most important part of any iris recognition is the matching process. From the last few years hamming distance was popularly used for iris template matching. This process is also a time consuming and need to replace with the technique which is efficient and less complex.

To overcome the identified problems of conventional iris recognition system, this work proposes following modification on conventional iris recognition system.

1) Development of highly efficient edge detection for iris using Morphological bridged canny edge detection instead of conventional canny edge detection with Hough transform.

2) In feature extraction, formation of feature matrix after preparation of rubber sheet model instead of using 2-D Gabor wavelet transform method using discrete wavelet transform.

3) For iris feature classification and matching process a highly efficient ANFIS classifier is proposed which leads to efficient solution for real time recognition.

The proposed methodology starts with very basic segmentation of iris with modified technique and uses 


\section{International Journal of Science and Research (IJSR) \\ ISSN (Online): 2319-7064 \\ Index Copernicus Value (2015): 78.96 | Impact Factor (2015): 6.391}

conventional method up rubber sheet model formation and ends with modified matching algorithm based on ANFIS Classifier. Figure (1) shows the complete proposed method with the help of flow chart.

\section{Experiments}

\section{a) Overview}

In this section In order to supply correct recognition of people, the foremost discriminating data gift in associate iris pattern should be extracted. Solely the numerous options of the iris should be encoded in order that comparisons between templates are often created. Most iris recognition systems build use of a band pass decomposition of the iris image to make a biometric guide.

\section{b) Methodology}

My work proposes a highly efficient real time iris recognition system based on simplest feature space of rubber sheet models discrete wavelet transform (DWT) feature, along with the efficient ANFIS classifier for iris image classification. The identified problems involve in traditional iris recognition system are:

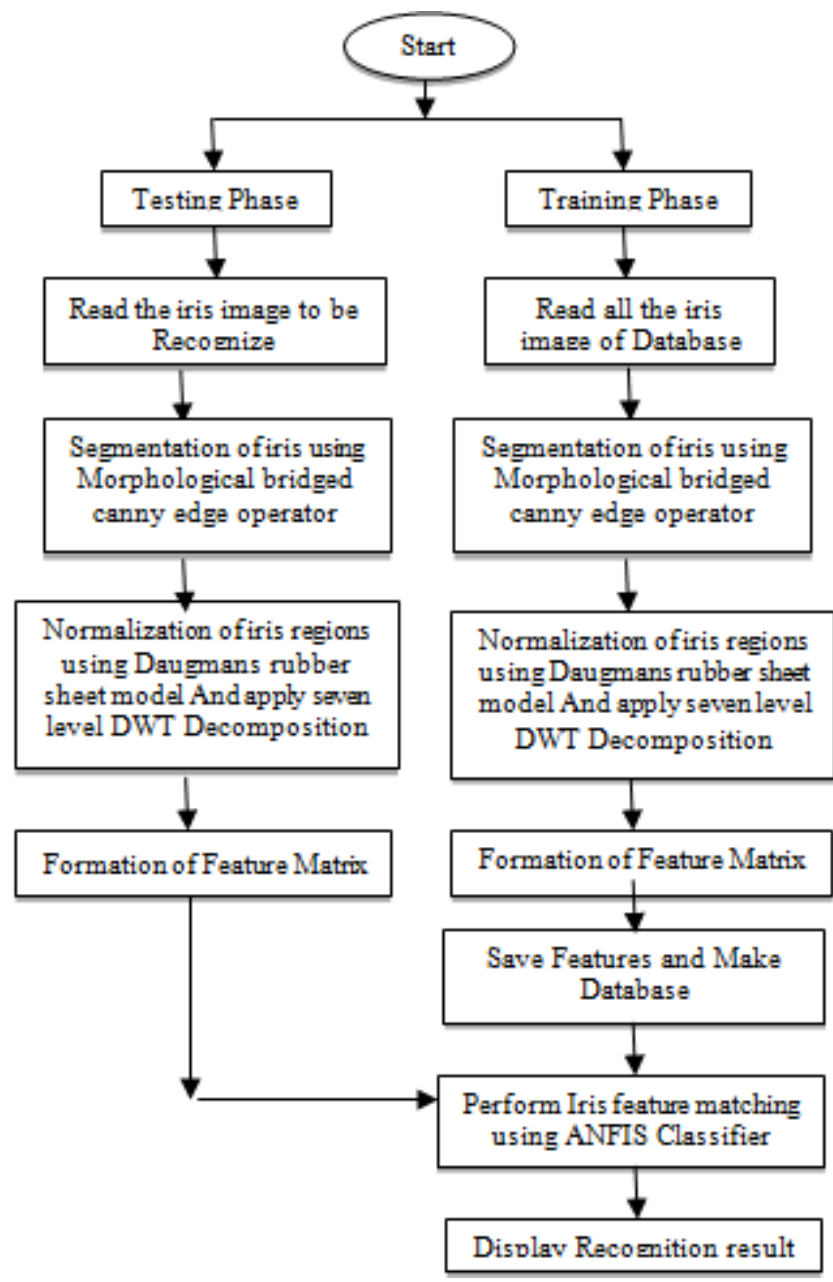

Figure 2: Process of Iris Recognition

1) The process is much easier than other to perform efficient iris recognition.

2) In the first step, that is for iris segmentation part mostly canny edge detector with Hough transform for circle detection has been employed. This process fails to avoid iris occlusion problem and not to draw complete circle and hence provides wrong information about pupil center coordinates and pupil radius.

3) In case of traditional system, lots of complex algorithms have been proposed by the researchers to extract features of the iris for recognition process, most of the time all these methods were very time consuming; hence an algorithm is required to overcome this problem.

4) The last but most important part of any iris recognition is the matching process. From the last few years hamming distance was popularly used for iris template matching. This process is also a time consuming and need to replace with the technique which is efficient and less complex.

5) To overcome the identified problems of conventional iris recognition system, this work proposes following modification on conventional iris recognition system.

6) Development of highly efficient edge detection for iris using Morphological bridged canny edge detection instead of conventional canny edge detection with Hough transform.

7) In feature extraction, formation of feature matrix after preparation of rubber sheet model instead of using 2-D Gabor wavelet transform method using discrete wavelet transform.

8) For iris feature classification and matching process a highly efficient ANFIS classifier is proposed which leads to efficient solution for real time recognition.

The proposed methodology starts with very basic segmentation of iris with modified technique and uses conventional method up rubber sheet model formation and ends with modified matching algorithm based on ANFIS Classifier.

\section{Results and Discussions}

In figure-3 we are selecting one image from the database and upload it for identification process.

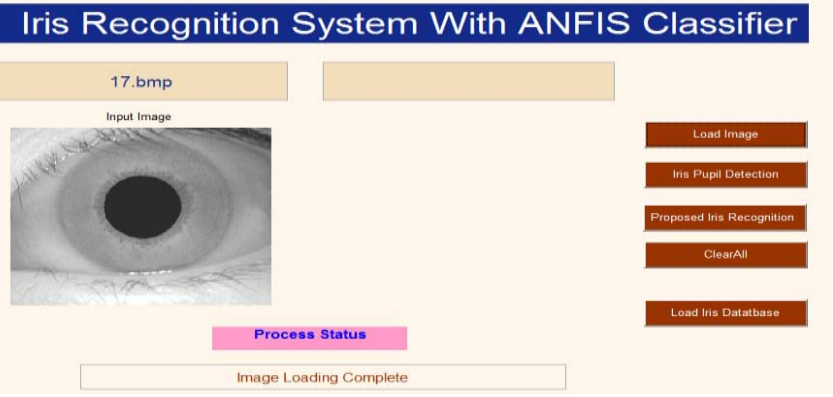

Figure 3: Image loading from database.

Then pupil is selected from that iris image for centre and radius localization (Figure-4). 


\section{International Journal of Science and Research (IJSR) ISSN (Online): 2319-7064}

Index Copernicus Value (2015): 78.96 | Impact Factor (2015): 6.391

Iris Recognition System With ANFIS Classifier

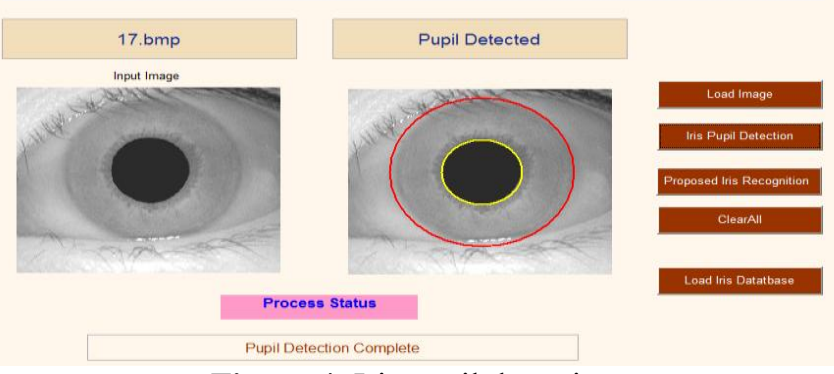

Figure 4: Iris pupil detection.

DAUGHMAN RUBBER SHEET MODEL - The homogenous rubber sheet model devised by daughman remaps each point within the iris region to a pair of polar coordinates $(r, \theta)$ where $r$ is on the interval $[0,1] \& \Theta$ is the angle $[0,2 \pi]$.

Finally in Figure-5 after Daughman rubber sheet model and discrete wavelet transform we apply ANFIS classifier to get the final result whether iris is matching from database or not.
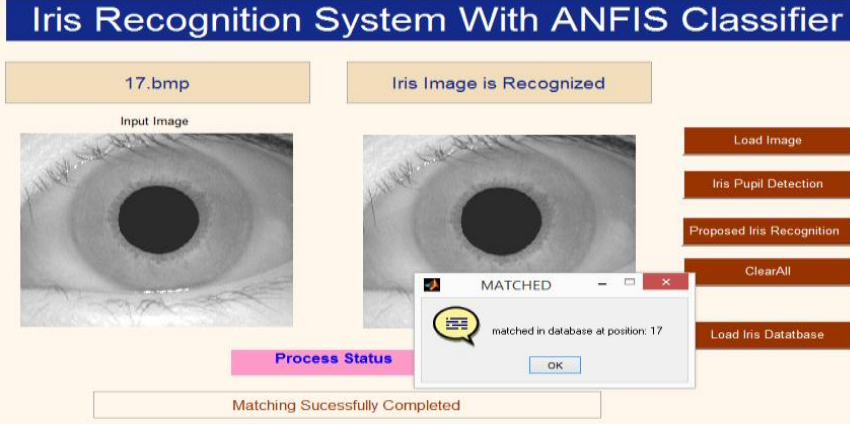

Figure 5: Image identification from database.

Figure-6 indicates the clusters and graph image of one iris image identification.

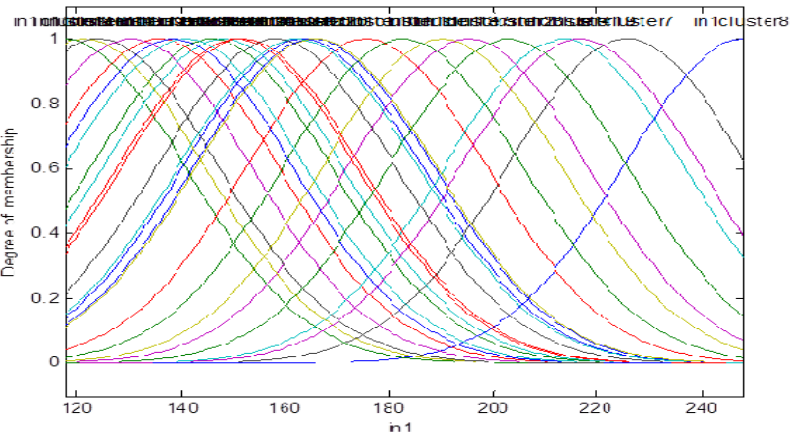

Figure 6: Clustered graph of one iris

\section{Conclusion and Future Research}

After understanding my has bestowed an iris recognition system that was tested victimization 2 databases of gray scale eye pictures so as to verify the claimed performance of iris recognition technology. Iris recognition is one in all handiest technique for identification of person. This paper provides review on varied existing strategies projected by totally different researchers from time to time for iris recognition.
The main functions of the bestowed algorithms are enhancing accuracy and reducing procedure time. The most plans were to extract a lot of correct region among image regions. Firstly, AN automatic segmentation algorithmic rule was bestowed, which might localize the iris region from a watch image and isolate palpebral, hair and reflection areas. Automatic segmentation was achieved through the utilization of the circular Hough remodel for localizing the iris and pupil regions, and also the linear Hough remodel for localizing occluding eyelids. Thresholding was extensively utilized for uninflected eyelashes and reflections. There are varied techniques that may be used for this purpose. Overall segmentation accuracy of these techniques has been analyzed. Higher the segmentation rate, therefore higher is its performance the unified framework has the very best segmentation rate and conjointly has highest performance.

The system conferred during this publication was ready to perform accurately, but there are still varieties of problems which require being self-addressed. 1st of all, the automatic segmentation wasn't excellent, since it couldn't with success phase the iris regions for all of the attention pictures within the 2 databases. So as to enhance the automated segmentation rule, a lot of elaborate lid and cilium detection system might be enforced. The cluster rule will be extended to localize exactly the iris region. Besides, analyzing alternative image characteristics such as intensity distribution or the luminosity part in YUV color area may conjointly improve the results. AN improvement may even be created within the speed of the system.

\section{Acknowledgment}

I would like to thank my guide Ms. Preeti Singh, Department of Computer Science and Engineering for her immense support and enlightened guidance for my project which I have developed as a M. Tech. student. I am very grateful for the inspiring discussions with all my faculty members. Their valuable support and path-guiding suggestions have helped me to develop this project. I am thankful to my HOD Mr. Bhanu Pratap Singh, Department of Computer Science and Engineering for giving thoughtful suggestions during my work.

\section{References}

[1] Abbasi Asima Akber, Khan M.N.A., Khan Sajid Ali (2013) "A Critical Survey of Iris Based Recognition Systems", A Critical Survey of Iris Based Recognition Systems, PP. 663-668.

[2] Pradnya M. Shende, Dr.Milind V. Sarode, Prof. Mangesh M. Ghonge (April 2014) " A Survey Based on Fingerprint, Face and Iris Biometric Recognition System, Image Quality Assessment and Fake Biometric", International Journal of Computer Science Engineering and Technology( IJCSET) Vol 4, Issue 4,PP. 129-132

[3] Dolly Choudhary, Shamik Tiwari, Ajay Kumar Singh, (Nov 2012) " A Survey: Feature Extraction Methods for Iris Recognition", International Journal of Electronics Communication and Computer Technology (IJECCT), Volume 2 Issue 6. 


\section{International Journal of Science and Research (IJSR) \\ ISSN (Online): 2319-7064 \\ Index Copernicus Value (2015): 78.96 | Impact Factor (2015): 6.391}

[4] Amrata Gupta1, Sachin Mahajan, (Jan 2014) "A Survey: IRIS Recognition With Different Techniques", International Journal of Software and Hardware Research in Engineering, Volume. 3, Issue 1, pp. 14-17.

[5] Arulalan.V, Balamurugan.G, Premanand. V, (Feb 2014) "A Survey on Biometric Recognition Techniques", International Journal of Advanced Research in Computer and Communication Engineering. Vol. 3, Issue 2, PP.5708-5711.

[6] Nirali M. Bhagwagar, Yagnik A. Rathod, (Apr 2015) "A Survey On Iris Recognition For Authentication", International Journal of Technical Research and Applications, Volume 3, Issue 2, PP. 148-151.

[7] Senbhaga S, (May 2013) " A Survey on Iris Segmentation using Distantly Acquired Face Images", International Journal of Scientific \& Engineering Research, Volume 4, Issue 5, PP. 118-123.

[8] Sarabjeet Kaur, (April 2015) "A Survey On Secure A Biometric Authentication", International Journal of Advanced Trends in Computer Applications (IJATCA) Volume 1, Number 4, PP. 24-27.

[9] Nidhi Manchanda, Oves Khan, Rishita Rehlan and Jyotika Pruthi, (2013) " A Survey: Various Segmentation Approaches to Iris Recognition", International Journal of Information and Computation Technology. Volume 3, Number 5 (2013), pp. 419-424.

[10] Yao-Hong Tsai, (2014) "A Weighted Approach to Unconstrained Iris Recognition", International Journal of Computer, Control, Quantum and Information Engineering Vol: 8, No:1 ,PP. 30-33.

[11] Gourav Sachdeva, Dr. Bikrampal Kaur (April 2015) "An Analytical Study On Iris Recognition System: A Survey", International Journal of Engineering Research and General Science Vol: 3, PP. 849-853.

[12] Lokesh Sharma, Gautam Thakur (August 2014) "An overview and examination of iris recognition Algorithms", International Journal of Advance Research in Computer Science and Management Studies Vol: 2, PP. 152-160

[13]Chandrakant D Patel, Sanket Trivedi, Sanjay Patel, (January 2012) "Biometrics in IRIS Technology: A Survey", International Journal of Scientific \& Research Publications Vol: 2, .PP 1-5.

[14] Samarth Bharadwaj, Mayank Vatsa and Richa Singh, (Jan 2014) "Biometric quality: a review of fingerprint, iris, and face", Springer, PP. 1-28

[15] Mayank Vatsa, Richa Singh, P. Gupta, (2004) "Comparison of iris Recognition Algorithms", IEEE. Vol. 3, Issue 2, PP.354-358.

[16] Nicolaie Popescu-Bodorin, "Exploring New Directions in Iris Recognition", IEEE, PP. 1-8.

[17] Amer Al-Rahayfeh And Miad Faezipour, (November 2013) "Eye Tracking and Head Movement Detection: A State-of-Art Survey", IEEE Vol:1 PP 2168-2372.

[18] Fabrizio Santini, Gabriel Redner, Ramon Iovin, and Michele Rucci, (2007) "EyeRIS: A general-purpose system for eye-movement-contingent display control", Boston University, Boston, Massachusetts, PP. 350-364.

[19]P.M. Agarkar, S.N. Talbar, (January 2013) "Fast Iris Searching Methods, Databases and Complex Patterns", MIT International Journal of Computer Science \& Information Technology Vol. 3, PP. 22-25. 